\title{
MULTIPHASE FLOW ANALYSIS OF MIST TRANSPORT BEHAVIOR IN AEROSOL JET® SYSTEM
}

\author{
JAMES Q. FENG \\ Optomec. Inc., USA.
}

\begin{abstract}
The Aerosol Jet ${ }^{\circledR}$ direct-write technology enables microscale printing of functional inks on various substrates with complex shapes, which is of great interest in many applications. It deposits the ink material in a form of high-speed collimated mist stream of fine droplets with diameters typically ranging from 1 to 5 microns, through a deposition nozzle connected via mist transport pathways to a liquid atomizer. Because the liquid atomization process often produces droplets with a wide size distribution having diameters much larger than 5 microns, removal of large ink droplets becomes the primary task of mist transport pathway design in the Aerosol Jet ${ }^{\circledR}$ system development, especially for printing fine features down to the 10 micron range. In the same time, the mist transport pathways must allow smaller droplets to pass through for desired ink material throughput. Therefore, it is important to understand the physical principles for mist transport pathway design based on multiphase mist flow analysis. With simplified models of particles flowing with a carrier gas in straight tubes of various inclination angles, convenient analytical formulas can be derived (even with particle inertia effect included to describe trajectories of large droplets), for calculating the rate of removal of droplets of given sizes due to gravitational sedimentation as a function of tube diameter and length. Rather intricate effect of particle inertia on gravitational wall deposition is revealed by the analytical results. For mist pathways of more complicated geometries, an OpenFOAM ${ }^{\circledR}$ lagrangian solver is used for the mist flow simulation, to gain insights into the wall deposition behaviour of ink droplets during mist transport from the liquid atomizer to the ink deposition nozzle. Applications of such mist flow analysis for mist transport channel design are illustrated with practical examples.

Keywords: Aerosol Jet@, gravitational sedimentation, mist of microdroplets, mist transport, OpenFOAM® lagrangian solver, particle-laden flow, wall deposition
\end{abstract}

\section{INTRODUCTION}

The Aerosol Jet ${ }^{\circledR}$ direct-write technology enables high-resolution (e.g. down to 10 microns) conformal printing of functional inks on various substrates with complex shapes, which is of particular interest in additive manufacturing 3D electronics, sensors, to name a few. In the Aerosol Jet ${ }^{\circledR}$ system (as illustrated in Fig. 1), the functional ink material is aerosolized in a liquid atomizer, transported through channels and deposited on substrate in a form of collimated aerosol mist stream of microdroplets with high-speed jet flow [1]. To enable accurate control of fine feature material deposition, ink droplets of diameters ranging from 1 to 5 microns are found to be mostly suitable for Aerosol Jet ${ }^{\circledR}$ printing (under the ambient condition with nitrogen as the processing gas) [2]. This is because droplets of too small a size cannot be deposited by inertial impaction, whereas larger droplets of too much inertia would not follow the aerodynamic focusing flow in the converging channel of the deposition nozzle, and rather move across streamlines to deposit onto the channel wall resulting in nozzle clogging. Moreover, droplets of diameters greater than 5 microns may not be useful for printing 10 micron lines due to the spread factor of individual droplet impact on substrate [3].

But the liquid atomizer for ink mist generation usually produces droplets of wide size distribution having diameters much larger than 5 microns. For example, a Collison atomizer, even with effective large droplet removal mechanism implemented, still contains droplets as 
- Atomizer to aerosolize liquid ink $(1-1000 \mathrm{cp})$

- Microdroplets of $1-5 \mu \mathrm{m}$ ( ${ }^{\sim} 5 e 6$ per cc) that enable printing features as small as $10 \mu \mathrm{m}$

- Sheath gas around mist stream in converging nozzle channel to focus narrow aerosol beam

- High-speed jet flow ( $\left.{ }^{\sim} 50 \mathrm{~m} / \mathrm{s}\right)$ to keep aerosol beam collimated for $2-5 \mathrm{~mm}$

- Able to print on substrates of complex shapes

Figure 1: Schematic of Aerosol Jet@ direct-write working principle.

large as 20, 30 and even 40 microns in its mist output [4]. Thus, mechanisms for removing large ink droplets are required before the mist enters the deposition nozzle of the Aerosol Jet ${ }^{\circledR}$ system. This becomes one of the primary functions of the mist transport pathways between the liquid atomizer and deposition nozzle. Actually, not only must the mist transport pathways be able to remove undesirable large droplets but they also must allow the droplets less than 5 microns to pass through as much as possible, when considering the ink throughput and equipment productivity. To accomplish such conflicting functionalities, it is important to understand the basic physical principles for mist pathway design based on multiphase mist flow analysis.

\section{PARTICLE-LADEN LAMINAR FLOW IN A STRAIGHT TUBE}

Among many possibilities, tubes of circular cross-section are the most commonly used channels for mist transport in the Aerosol Jet ${ }^{\circledR}$ system, as schematically illustrated in Fig. 1. When flowing with a carrier gas through a tube, the mist droplets will always have a velocity component perpendicular to the gas flow streamlines, unless the gravity is absent or has its direction perfectly coincident with the tube axis. This leads to gravity-driven droplet deposition onto the tube wall, and the tube can serve as a 'passive filter' for effectively removing large droplets. Mathematical solutions of gravitational deposition of 'inertialess' particles from laminar flow in channels were derived by several authors [5, 6]. Ignoring the particle inertia effect (by setting the particle Stokes number to zero) certainly helps simplify mathematical derivations; but the results may be inaccurate for large particles with substantial mass, which are sometimes encountered in the Aerosol Jet ${ }^{\circledR}$ process. In what follows, general mathematical formulas are derived including the particle inertia effect with nonzero Stokes number, for examining the validity range of the theory for inertialess particles. Then the mathematical formulas are used for predicting the pass-through rate for particles of 
various sizes for given tube diameter and length with flow rate relevant to Aerosol Jet ${ }^{\circledR}$ printing. The analytical results can also be used for verifying numerical CFD models.

\subsection{General mathematical formulas}

The general mathematical formulas for particle trajectories including the particle inertia can be derived by considering the motion of a rigid spherical particle (as a reasonable approximation to the ink microdroplet) in a laminar gas flow within a straight tube. For a circular tube of radius $R$ with its axis aligned with the Cartesian $z$-axis which points in the direction of gas flow, and the vector of gravitational acceleration is in the $y z$-plane such that $g=g\left(e_{y} \sin \varphi+e_{z} \cos \varphi\right)$ with $g=9.81 \mathrm{~m} \mathrm{~s}^{-2}, e_{y}$ and $e_{z}$ denoting unit vectors in the direction of $y$-axis and $z$-axis, $\mathrm{f}$ the angle measured from $z$-axis to $y$-axis. If the laminar gas flow in the tube is assumed well-developed, it has only the $z$-component described by the Poiseuille parabolic profile with a mean velocity of magnitude $U$. With velocity, length and time, respectively, measured in units of $U, R$ and $R / U$, the governing equations for the motion of a particle of diameter $d$ and density $\rho$ can be written as

$$
\left\{\begin{array}{c}
\mathrm{St} \frac{\mathrm{d} u_{x}}{\mathrm{~d} t}=-u_{x} \\
\mathrm{St} \frac{\mathrm{d} u_{y}}{\mathrm{~d} t}=U_{s} \sin \varphi-u_{y} \\
\mathrm{St} \frac{\mathrm{d} u_{z}}{\mathrm{~d} t}=U_{s} \cos \varphi-u_{z}+2\left(1-x^{2}-y^{2}\right)
\end{array},\right.
$$

where $u_{x}=\mathrm{d} x(t) / \mathrm{d} t, u_{y}=\mathrm{d} y(t) / \mathrm{d} t$ and $u_{z}=\mathrm{d} z(t) / \mathrm{d} t$ denote $x$-, $y$ - and $z$-components of particle velocity,

$$
\mathrm{St}=\frac{\rho U C d^{2}}{18 \mu R}
$$

is the particle Stokes number (with $C$ denoting the Cuningham slip correction factor and $\mathrm{m}$ the viscosity of carrier gas) [7], and

$$
U_{s}=\frac{\rho g C d^{2}}{18 \mu U}=\mathrm{St} \frac{g R}{U^{2}}=\frac{\mathrm{St}}{\mathrm{Fr}^{2}} \text {, where Fr denotes the Froude number }
$$

is the dimensionless gravitational settling velocity of the particle. Thus, there are two dimensionless parameters appearing in (1), which is generally a set of nonlinear, nonhomogeneous differential equations. The value of St describes the ratio of particle relaxation time (i.e. the characteristic time for a particle to adjust in response to the change in surrounding gas flow) and the characteristic time of gas flow $R / U$, while that of $U_{s}$ the ratio of particle settling velocity and the characteristic velocity of gas flow $U$.

After some lengthy tedious derivations, the solution to eq. (1) for particle trajectory is obtained as 


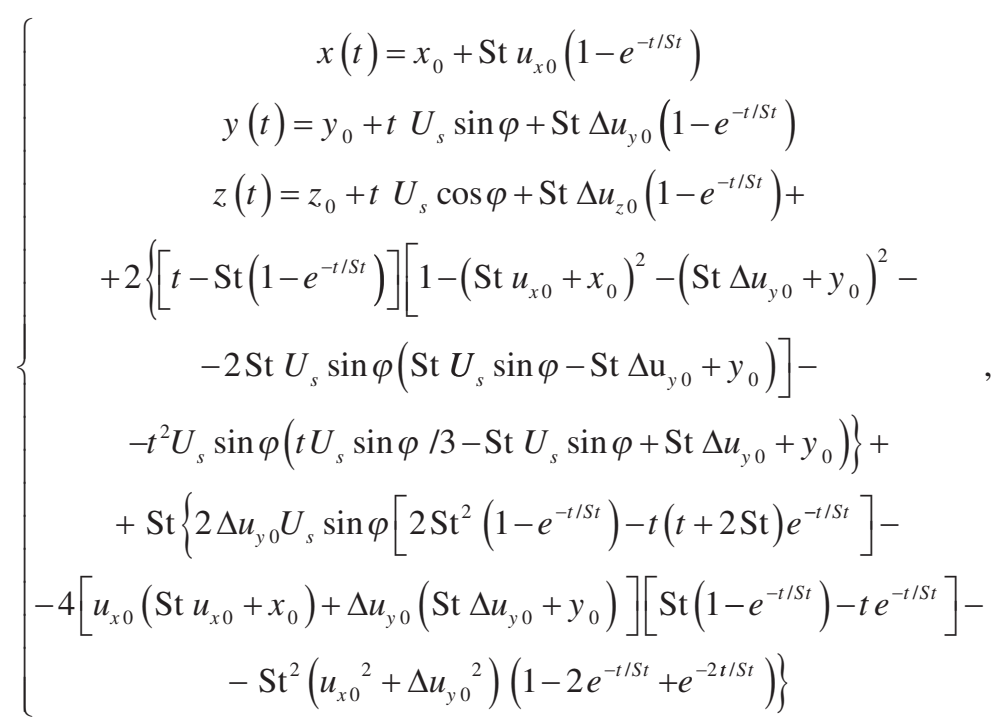

where $x_{0}=x(0), y_{0}=y(0)$ and $z_{0}=z(0)$ are the initial position of particle, and $\Delta u_{y 0}=u_{y 0}-U_{s} \sin \varphi$, $\Delta u_{z 0}=u_{z 0}-U_{s} \cos \varphi$, with $u_{x 0}, u_{y 0}$ and $u_{z 0}$ denoting the particle initial velocity components.

Noteworthy here is that the nonlinearity due to the term of $y^{2}$ in (1) yields another dimensionless parameter

$$
\Gamma=\mathrm{St} U_{s}=\frac{g}{R}\left(\frac{\rho C d^{2}}{18 \mu}\right)^{2}=\left(\frac{\mathrm{St}}{\mathrm{Fr}}\right)^{2}
$$

which is the ratio of the particle relaxation time and characteristic time of gravitational sedimentation squared. While St increases with $U$ and $U_{s}$ decreases with $U$, the value of $\Gamma$ is independent of $U$ but strongly dependent on the particle size $d$ (to the $4^{\text {th }}$ power).

It is often convenient to assume that $u_{x 0}=\Delta u_{y 0}=\Delta u_{z 0}-2\left(1-x_{0}{ }^{2}-y_{0}{ }^{2}\right)=0$ for the particle to be initially at a mechanical equilibrium state in a fully developed laminar flow, to simplify the analysis with reduced number of variables. Without loss of generality, setting $z_{0}=0$ is also desirable. Thus (4) becomes much cleaner as

$$
\left\{\begin{array}{c}
x(t)=x_{0} \\
y(t)=y_{0}+t U_{s} \sin \varphi \\
z(t)=t U_{s} \cos \varphi+2\left\{t\left(1-x_{0}{ }^{2}-y_{0}{ }^{2}\right)-2\left[t-\mathrm{St}\left(1-e^{-t / S t}\right)\right] \times\right. \\
\left.\times \Gamma \sin \varphi\left(\Gamma \sin \varphi+y_{0}\right)-t^{2} U_{s} \sin \varphi\left(t U_{s} \sin \varphi / 3-\Gamma \sin \varphi+y_{0}\right)\right\}
\end{array}\right.
$$

For consistent analysis, it is also assumed that $\sin \varphi<0$, indicating that the $y$-component of gravitational acceleration always points to the negative $y$-direction (e.g. $180^{\circ}<\varphi<360^{\circ}$, with $\varphi=270^{\circ}$ representing mist flow in a horizontal tube, $\varphi<270^{\circ}$ uphill mist flow and $\varphi>270^{\circ}$ downhill mist flow). Thus, gravitational settling can only cause particles to deposit on the lower half of the tube wall $y=-\left(1-x^{2}\right)^{1 / 2}$. 
For a tube of length $L$, the value of pass-through rate or 'penetration' $P$ (defined as the fraction of particles with uniform concentration at inlet $z=0$ that have not deposited on the tube wall when arriving at $z=L$ ) is of great practical importance. If the particle inertia effect is negligible, i.e. when $\mathrm{St}=0$, (4) become much simpler that an analytical formula for $P$ can be obtained for mist flow in a horizontal tube [5] as

$$
P=1-\frac{2}{\pi}\left[(2 \varepsilon-1) \sqrt{\varepsilon(1-\varepsilon)}+\sin ^{-1} \varepsilon\right]
$$

where $\varepsilon=\left(0.375 U_{s} L\right)^{2 / 3}$. Later, (7) was found to be also applicable for uphill flow in an inclined tube of inertialess particles by replacing $\varepsilon=\left(0.375 U_{s} L\right)^{2 / 3}$ with $\varepsilon=\left(0.375 U_{s} L\right.$ $\sin \varphi)^{2 / 3} /\left(1+0.5 U_{s} \cos \varphi\right)$ when $\cos \varphi<0$, but for downhill flow when $\cos \varphi<0$ only approximate formula could be obtained [6].

With nonzero St in (4), it seems impossible to derive an analytical formula for $P$ like (7) due to the transcendental function forms. But the pass-through rate $P$ can be computed numerically without much difficulty by integrating over the inlet area above the curve that initiates the so-called 'limiting trajectories' (defined by those particles starting at $x_{0}$ and $y_{0}$ and arriving at $y=-\left(1-x^{2}\right)^{1 / 2}$ at $\left.z=L\right)$, with the available analytical formulas (4) as well as (6) implemented in a short program.

\subsection{Particle inertial effect}

The particle inertial effect on its moving trajectories can be easily examined with the derived formulas (6). Shown in Fig. 2 is the contrast between trajectories of $\mathrm{St}=0.44$ (solid) and

\section{horizontal tube $(\mathrm{St}=\mathbf{0 . 4 4 )}$}

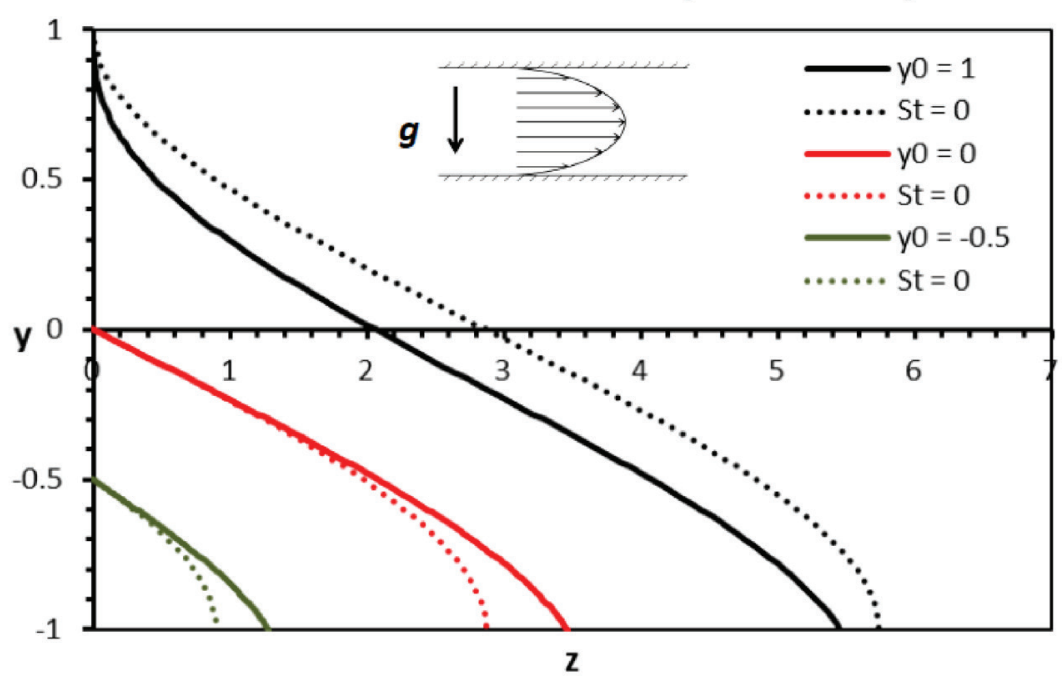

Figure 2: Particle trajectories in a horizontal tube $\left(\varphi=270^{\circ}\right)$ of $R=1.5 \mathrm{~mm}$ with $Q=50 \mathrm{ccm}$ for $\mathrm{St}=0.44$ (solid) and $\mathrm{St}=0$ (dotted) with initial positions along the line $x_{0}=z_{0}=0$ at $y_{0}=1,0$ and -0.5 , computed with (6). 
$\mathrm{St}=0$ (dotted) corresponding to a particle of $d=30 \mu \mathrm{m}$ and $\rho=2 \mathrm{~g} / \mathrm{cc}$ in a flow of volumetric flow rate $Q=50 \mathrm{ccm}\left(U=0.118 \mathrm{~m} / \mathrm{s}, \mathrm{St}=0.44, U_{s}=0.465, \Gamma=0.205\right)$ in a horizontal tube of $R=1.5 \mathrm{~mm}$, which are realistic parameters for Aerosol Jet ${ }^{\circledR}$ printing. Although majority of ink droplets have $d<5 \mu \mathrm{m}$ in Aerosol Jet ${ }^{\circledR}$ printing, droplets of $d>30 \mu \mathrm{m}$ have been observed in the mist output from the Collison-type atomizers [4] and must be removed before they arrive at the deposition nozzle. Those large droplets are expected to move with trajectories significantly different from those predicted for inertialess particles, as illustrated in Fig. 2. When flowing at a rate of $50 \mathrm{ccm}$ in a horizontal tube of $R=1.5 \mathrm{~mm}$, those droplets of $d>30 \mu \mathrm{m}$ should be completely removed by gravitational sedimentation after traveling a length of $L \sim 5.4$ (or $8.1 \mathrm{~mm}$ ).

Because of $\Gamma$ in (6), the particle inertial effect at nonzero St is expected to strongly depend on the particle size. If $d$ is reduced to $15 \mu \mathrm{m}$ (with $\mathrm{St}=0.11, U_{s}=0.117, \Gamma=0.013$ ), the difference from the trajectories for $\mathrm{St}=0$ would become barely noticeable. Thus, it seems to be safe to treat particles as inertialess when $\Gamma<0.01$ as a criterion for rough estimate (for horizontal tube).

\subsection{Particle pass-through rate in a horizontal tube}

In a typical setting (cf. Fig. 1), there is always certain length of horizontal channel for mist transport from an atomizer to deposition nozzle in the Aerosol Jet ${ }^{\circledR}$ system. As suggested by (7), the pass-through rate of particles of a given size is determined by the tube radius $R$ and (dimensional) length $L^{*}(=L R)$ along with $U$ or the mist flow rate $Q\left(=\pi R^{2} U\right)$. Listed in Table 1 are computed numerical values of particle pass-through rate $P$ in a horizontal tube of $R=1.5 \mathrm{~mm}$ and (dimensional) length $L^{*}=50 \mathrm{~mm}$ at various flow rate $Q$ for $d=1,3,5,8$ and $12 \mu \mathrm{m}$ with $\rho=2 \mathrm{~g} / \mathrm{cc}$, with that calculated according to (7) for inertialess particles given in parentheses for comparison.

Even for particles of $d=12 \mu \mathrm{m}$ at $Q=400 \mathrm{ccm}\left(U_{s}=0.0094\right.$ and $\left.\Gamma=0.0054\right)$, the computed value of $P$ is only less than one percent greater than that predicted with (7), which is consistent with our suggested criterion of $\Gamma<0.01$ for negligible inertia effect. If $d$ is increased to $30 \mu \mathrm{m}$ (with $Q=400 \mathrm{ccm}, \mathrm{St}=3.512, U_{s}=0.0581, \Gamma=0.204$ ), the computed value of $P$ is 0.190 (for $L^{*}=50 \mathrm{~mm}$ ) whereas that predicted by (7) would be 0.134 , indicating a significant inertia effect when $\Gamma>0.01$. With $d=20 \mu \mathrm{m}\left(\mathrm{St}=1.565, U_{s}=0.0581\right.$, $\Gamma=0.204$ ), the computed $P$ is 0.563 and that of (7) is 0.536 . Thus, the inertia effect for particles of $d<20 \mu \mathrm{m}$ is not expected to be overly important (because $\Gamma$ gets close to 0.01 ). For complete removal of droplets larger than $20 \mu \mathrm{m}$ at $Q=400 \mathrm{ccm}$, either increasing the tube

Table 1: Computed values of $P$ in a horizontal tube of $R=1.5 \mathrm{~mm}$ and (dimensional) length $L^{*}=50 \mathrm{~mm}$ at various $Q$ for particles of $d=1,3,5,8$ and $12 \mu \mathrm{m}$ with $\rho=2 \mathrm{~g} / \mathrm{cc}$.

\begin{tabular}{llllll}
\hline$Q(\mathrm{ccm})$ & $d=1$ & 3 & 5 & 8 & $12(\mu \mathrm{m})$ \\
\hline 25 & 0.975 & 0.809 & 0.527 & $0.061(0.060)$ & 0 \\
50 & 0.987 & 0.902 & 0.746 & $0.425(0.424)$ & 0.015 \\
100 & 0.994 & 0.950 & 0.868 & $0.686(0.685)$ & $0.373(0.369)$ \\
200 & 0.997 & 0.975 & 0.932 & $0.835(0.834)$ & $0.655(0.651)$ \\
400 & 0.998 & 0.987 & 0.966 & $0.916(0.915)$ & $0.820(0.816)$ \\
\hline
\end{tabular}


length to $L^{*}=150 \mathrm{~mm}$ (with $R=1.5 \mathrm{~mm}$ ) or increasing $R$ to $4.5 \mathrm{~mm}$ (with $L^{*}=50 \mathrm{~mm}$ ) can be effective.

A tube of $L^{*}=50 \mathrm{~mm}$ is not very long, but almost half of the $5 \mu \mathrm{m}$ droplets will be lost by deposition on the wall (while no droplets of $d>8 \mu \mathrm{m}$ can pass through) at low flow rate of $Q$ $=25 \mathrm{ccm}$. If longer tube must be used, e.g. due to hardware configuration limitations with a particular application, a tube of smaller $R$ should be used for the low flow rate fine-feature printing. As suggested by (7), the value of the dimensionless variable $\varepsilon$ is mostly determined by $d^{2} \mathrm{C} R L^{*} / Q$, which is especially accurate for the case of horizontal tube when particle inertia is negligible. Thus, the value of $P$ is expected to remain the same as long as the value of $d^{2}$ C $R L^{*} / Q$ is fixed. This fact is illustrated by Table 2 where the values of $P$ for a given particle size remains the same as that in Table 1 when $R$ and $Q$ are both reduced by a factor of 0.5 (with $L^{*}$ fixed at $50 \mathrm{~mm}$ ). A slight change of $P$ for $d=12 \mu \mathrm{m}$ can be attributed to the inertia effect. For the same $R$ and $L^{*}$ in Table 2, particles of $d=8 \mu \mathrm{m}$ has $P \sim 0.01$ at $Q=11 \mathrm{ccm}$, while those of $d=5 \mu \mathrm{m}$ will have $P$ become $\sim 0.01$ when $Q$ is reduced to $4.3 \mathrm{ccm}$ $\left(\sim 11 *(5 / 8)^{2} \mathrm{ccm}\right)$. Such a trend of the 'cut-off' droplet size decreasing with reducing $Q$ has been taken advantage of in the Aerosol Jet ${ }^{\circledR}$ printing practice to enable finer feature print with smaller droplets by reducing the mist flow rate $Q$ using the same mist-transport hardware setting.

\subsection{Particles moving uphill in a near-vertical tube}

For convenience of containing liquid ink in the atomizer, the generated mist is usually extracted in an uphill flow channel (as illustrated in Fig. 1). When using an ultrasonic atomizer in the Aerosol Jet ${ }^{\circledR}$ system, the mist first enters a near-vertical pick-up tube in the atomizer chamber, moving upward for a length about $60 \mathrm{~mm}$ before bending to a horizontal channel. The pick-up tube is usually set at about $10^{\circ}$ off vertical (for good reasons), with a radius $R=3 \mathrm{~mm}$.

As shown in Fig. 3 with a flow rate of $50 \mathrm{ccm}(U=0.029 \mathrm{~m} / \mathrm{s})$, it is difficult for a particle of $d=30 \mu \mathrm{m}$ to move with the upward gas flow due to the fact that $U_{s} \cos \varphi=-1.831$ while the peak gas velocity is 2 ; only those particles located around the tube center can be pushed upward by the gas flow. But the particles also move laterally at the same time, due to the nonzero $U_{s} \sin \varphi$; as soon as getting outside the central region of the tube, they will move downward against the gas flow. In fact, particles of $d=25 \mu \mathrm{m}\left(U_{s} \cos \varphi=-1.273\right)$ can only penetrate a short length $(\sim 1.5 \mathrm{~mm})$, whereas those of $d=20 \mu \mathrm{m}\left(U_{s} \cos \varphi=-0.816\right)$ can penetrate about $21 \mathrm{~mm}$. Particles moving uphill in a near-vertical tube generally exhibit rather complicated trajectories, with downward motion near the tube wall and upward motion when in the tube centre.

Table 2: As table 1 but for $R=0.75 \mathrm{~mm}$ and (dimensional) length $L^{*}=50 \mathrm{~mm}$.

\begin{tabular}{llllll}
\hline$Q(\mathrm{ccm})$ & $d=1$ & 3 & 5 & 8 & $12(\mu \mathrm{m})$ \\
\hline 5 & 0.939 & 0.560 & 0.066 & 0 & 0 \\
10 & 0.969 & 0.765 & 0.439 & 0 & 0 \\
25 & 0.987 & 0.902 & 0.746 & $0.425(0.424)$ & $0.016(0.015)$ \\
50 & 0.994 & 0.950 & 0.868 & $0.686(0.685)$ & $0.377(0.369)$ \\
\hline
\end{tabular}




\section{near-vertical tube (uphill)}

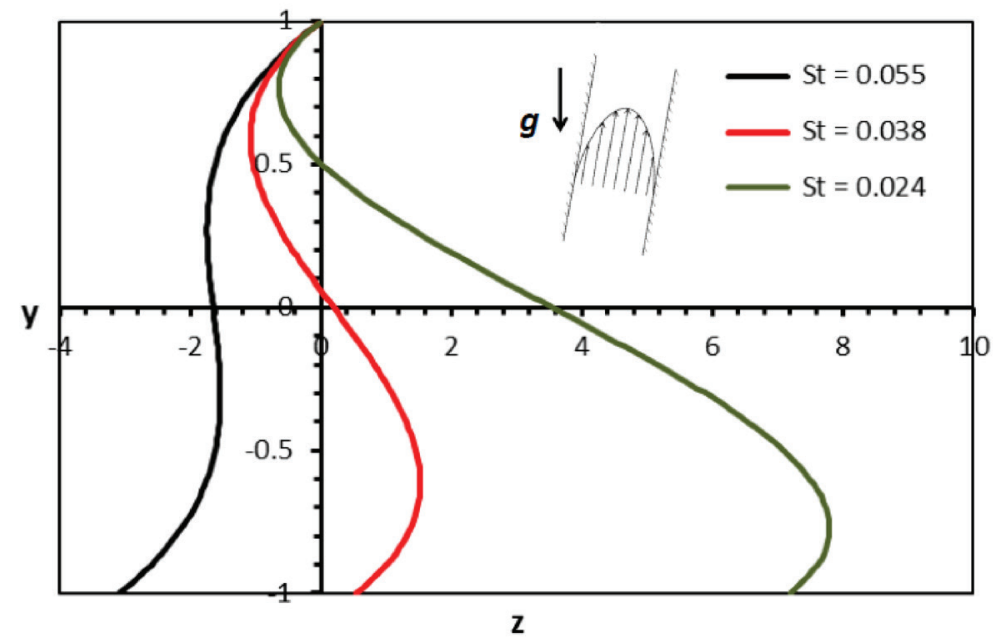

Figure 3: Particle trajectories in a near-vertical tube $\left(\varphi=190^{\circ}\right)$ of $R=3 \mathrm{~mm}$ with $Q=50 \mathrm{ccm}$ for $d=30 \mu \mathrm{m}(\mathrm{St}=0.055), 25 \mu \mathrm{m}(\mathrm{St}=0.038)$ and $20 \mu \mathrm{m}(\mathrm{St}=0.024)$ with initial positions along the line $x_{0}=z_{0}=0$ at $y_{0}=1$, computed with (6).

The computed values of $P$ for $R=3 \mathrm{~mm}$ and $L^{*}=60 \mathrm{~mm}$ are given in Table 3 with various flow rate $Q$. Larger particles are more difficult to carry by the gas flow and have lower passthrough rate $P$. With reducing $Q$, for printing finer features with the Aerosol Jet ${ }^{\circ}$ process, the pick-up tube naturally removes undesirable droplets of sizes larger than the print feature size, serving as a passive filter according to the mist flow rate $Q$ similar to the situation of mist flow in horizontal tube.

The comparison with the inertialess result (in parentheses) in Table 3 appears quite interesting, with (7) overestimate $P$ at lower $Q$ while underestimate $P$ at higher $Q$. This is because each of the three parameters St, $U_{s}$ and $\Gamma$ varies with $\Theta$ or $U$ differently, with St proportional to $U$, whereas $U_{s}$ inversely proportional to $U$ and $\Gamma$ independent of $U$.

Table 3: As table 1 but for $R=3 \mathrm{~mm}$ and $L^{*}=60 \mathrm{~mm}$ for uphill flow $\left(\varphi=190^{\circ}\right)$.

\begin{tabular}{llllll}
\hline$Q(\mathrm{ccm})$ & $d=1$ & 3 & 5 & 8 & $12(\mu \mathrm{m})$ \\
\hline 5 & 0.9479 & 0.572 & 0 & 0 & 0 \\
10 & 0.9738 & 0.787 & 0.416 & 0 & 0 \\
25 & 0.9895 & 0.915 & 0.768 & $0.404(0.409)$ & 0 \\
50 & 0.9947 & 0.957 & 0.885 & $0.706(0.707)$ & $0.335(0.340)$ \\
100 & 0.9974 & 0.979 & 0.942 & $0.854(0.854)$ & $0.671(0.672)$ \\
200 & 0.9986 & 0.989 & 0.971 & $0.927(0.927)$ & $0.837(0.837)$ \\
400 & 0.9993 & 0.995 & 0.985 & $0.964(0.963)$ & $0.919(0.918)$ \\
\hline
\end{tabular}




\subsection{Particles moving downhill in a near-vertical tube}

Before entering the deposition nozzle, the mist typically flows downward in a near-vertical mist feeding tube (cf. Fig. 1). Although it is theoretically desirable to have the mist feeding tube connecting the deposition nozzle oriented vertically for symmetric mist distribution, slight imperfection always exists in reality. The formulas (4) can be used for evaluating the rate of wall deposition of particles moving down in a near vertical tube, which can be useful for setting the mist transport tube design tolerance.

Table 4 shows that some particles will always deposit on the tube wall even with fairly high pass-through rate $P$ in a near-vertical tube with downhill flow. At a given flow rate $Q$, larger particles are more likely to deposit on the tube wall. To reduce the amount of ink droplet deposition onto the near-vertical mist feeding tube wall in a downhill flow before entering the deposition nozzle, it is important to consider keeping the tube as vertical as possible and removing large droplets through the uphill flow in atomizer pick-up tube and the flow in horizontal portion of the mist transport channel prior to entering the mist feeding tube in the Aerosol Jet ${ }^{\circledR}$ system.

When considering the particle 'precipitation efficiency' $E=1-P$, the rate of particle wall loss $E$ is also significantly affected by the off-vertical tilt angle (described by the value of $\mathrm{f}$ ). For example, the value of $E$ for a $45^{\circ}$ tilt tube $\left(\varphi=315^{\circ}\right)$ would become about 5 times that with $\varphi=350^{\circ}$. On the other hand, the value of $E$ can be reduced by a factor of 0.4 for $\varphi=355^{\circ}$ from that with $\varphi=350^{\circ}$. Thus, keeping $\varphi$ as close as possible to $360^{\circ}$ (perfectly vertical) can be quite effective for minimizing the amount of particle wall deposition with the downhill flow in the mist feeding tube.

\section{MIST FLOW IN A TURNING CHAMBER OF COMPLEX SHAPE}

In reality, the mist transport pathways in Aerosol Jet ${ }^{\circledR}$ system often consist of portions with complex shapes wherein the particles undergo curvilinear motions, for which the straight tube model becomes inapplicable. For analysing mist flow in passages of complex shapes, the OpenFOAM ${ }^{\circledR}$ CFD Toolbox (cf. www.openfoam.com/documentation/) is used to construct numerical models. Within the framework of OpenFOAM®, a lagrangian solver is implemented by adding a basicKinematicCloud class to the transient incompressible flow solver pimpleFoam (using a merged PISO-SIMPLE algorithm for solving the transient Navier-Stokes equations), which allows simulation of various particle-flow interactions.

Table 4: As table 1 but for $R=1 \mathrm{~mm}$ and $L^{*}=50 \mathrm{~mm}$ for downhill flow $\left(\varphi=350^{\circ}\right.$ without data in parentheses because no exact formula for $\mathrm{St}=0$ is available)

\begin{tabular}{llllll}
\hline$Q(\mathrm{ccm})$ & $d=1$ & 3 & 5 & 8 & $12(\mu \mathrm{m})$ \\
\hline 5 & 0.9882 & 0.908 & 0.762 & 0.464 & 0.058 \\
10 & 0.9941 & 0.953 & 0.876 & 0.706 & 0.414 \\
25 & 0.9976 & 0.981 & 0.949 & 0.875 & 0.735 \\
50 & 0.9988 & 0.990 & 0.974 & 0.936 & 0.861 \\
100 & 0.9994 & 0.995 & 0.987 & 0.967 & 0.929 \\
200 & 0.9997 & 0.998 & 0.993 & 0.984 & 0.964 \\
400 & 0.9999 & 0.999 & 0.997 & 0.992 & 0.982 \\
\hline
\end{tabular}


To verify correctness of the lagrangian solver implementation, mist flow in a circular horizontal tube of $R=1.5 \mathrm{~mm}$ and (dimensional) length $L^{*}=50 \mathrm{~mm}$ is computed at $Q=100 \mathrm{ccm}$, to compare with the corresponding analytical results in Table 1. For particles of $d=1,3,5$ and $8 \mu \mathrm{m}$ with $\rho=2 \mathrm{~g} / \mathrm{cc}$, the OpenFOAM ${ }^{\circledR}$ computations yield $P=0.989,0.918,0.818$ and 0.621 , respectively (which are comparable to $0.994,0.950,0.868$ and 0.686 in Table 1 ). The slight reduction of computed $P$ seems to be a result of using the patch injection model for particle introduction to a plug (uniform) flow at the tube inlet in the OpenFOAM $®$ simulation, whereas the analytical formulas are derived by assuming the fully-developed parabolic flow throughout the entire tube.

As an example, the mist flow turning chamber in which the horizontal channel flow turns into downward vertical channel flow may take a configuration, as shown in Fig. 4, where the ink material deposited due to large droplet removal in the horizontal channel and chamber wall is prevented to flow downstream to the deposition nozzle, by having the vertical mist feeding tube inlet protruding above the chamber floor. The immediate engineering design question is by how far the protrusion may go before losing too much desirable small mist droplets (e.g. those with $d$ in the range of 1 to $5 \mu \mathrm{m}$ ) due to inertial impaction when particles undergo curvilinear motion [7].

The mist flow turning chamber shown in Fig. 4 consists of a vertical downstream tube of inner radius $1.5 \mathrm{~mm}$ concentrically positioned in a cylindrical chamber of radius $3.5 \mathrm{~mm}$ and height $9.0 \mathrm{~mm}$, with a (de-featured) straight rectangular horizontal channel having a height of $3.0 \mathrm{~mm}$ and a width of $5.0 \mathrm{~mm}$ attached to the chamber side wall. The vertical tube has its inlet protruding out $2.0 \mathrm{~mm}$ above the floor of the chamber and lower surface of the rectangular horizontal channel. With a mist flow rate of $Q=120 \mathrm{ccm}$ for particles of density $\rho=2$ $\mathrm{g} / \mathrm{cc}$ and $d$ ranging from 1.0 to $5.5 \mu \mathrm{m}$ injected with a Rosin-Rammler distribution from the

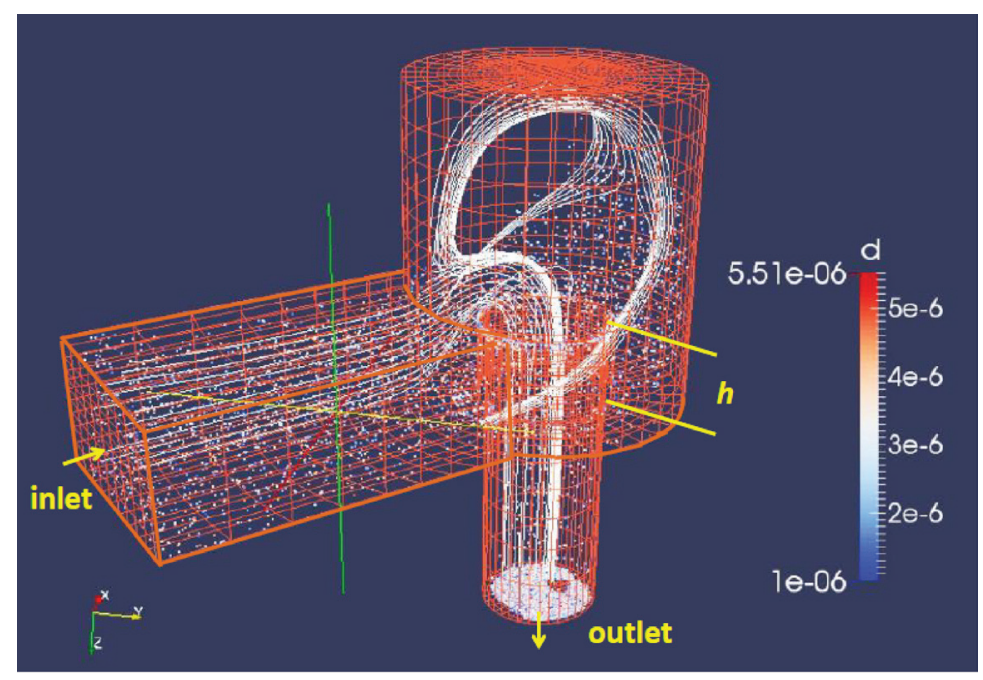

Figure 4: Mist flow of $Q=120 \mathrm{ccm}$ simulated with OpenFOAM ${ }^{\circledR}$ for a mist flow turning chamber wherein the mist particles move (left to right) from a horizontal channel to a vertical (downward flow) mist feeding tube, with particles and carrier gas streamlines displayed in a snapshot with ParaView (a multi-platform data analysis package). The 3D mesh is generated with the blockMesh utility included in the OpenFOAM ${ }^{\circledR}$ package. 
Table 5: Computed values of $P$ at various mist flow rate $Q$ for the mist flow turning chamber shown in Fig. 4 with the vertical tube protrusion of $h=1,2,3$ and $4 \mathrm{~mm}$ above the chamber floor.

\begin{tabular}{|c|c|c|c|c|}
\hline \multirow[b]{2}{*}{$Q(\mathrm{ccm})$} & \multicolumn{4}{|c|}{ Vertical tube protrusion $h$} \\
\hline & $1(\mathrm{~mm})$ & $2(\mathrm{~mm})$ & $3(\mathrm{~mm})$ & $4(\mathrm{~mm})$ \\
\hline 30 & 0.891 & 0.862 & 0.852 & 0.814 \\
\hline 60 & 0.946 & 0.930 & 0.922 & 0.917 \\
\hline 120 & 0.962 & 0.958 & 0.955 & 0.943 \\
\hline 240 & 0.977 & 0.970 & 0.969 & 0.952 \\
\hline 360 & 0.978 & 0.973 & 0.970 & 0.949 \\
\hline 480 & 0.977 & 0.968 & 0.965 & 0.937 \\
\hline
\end{tabular}

inlet patch (of the rectangular horizontal channel), this configuration of a mist flow turning chamber allows a particle mass pass-through rate $P$ of $95.8 \%$ (which is quite acceptable) despite some irregularities of the flow field in the chamber.

For convenience of comparison, Table 5 shows the computed values of pass-through rate $P$ at various mist flow rate $Q$ for the mist flow turning chamber configuration in Fig. 4 with the vertical tube protrusion of $h=1,2,3$ and $4 \mathrm{~mm}$ above the chamber floor. In a wide range of $Q$ (from 30 to $480 \mathrm{ccm}$ ), the value of $P$ seems to be rather insensitive to the vertical tube protrusion $h$, though with a gradual declining trend. The least variation of $P$ appears between $h=2$ and $3 \mathrm{~mm}$. The general trend of gradually reduced $P$ with decreasing $Q$ correlates to the increased time for gravitational sedimentation of particles prior to entering the vertical mist feeding tube. There is no noticeable inertial impaction loss of particles even at $Q=360 \mathrm{ccm}$, but at $Q=480 \mathrm{ccm}$ the sign of decreasing $P$ due to impaction loss emerges. Noticeable inertial impaction of particles on the chamber wall seems only to appear at $Q>480 \mathrm{ccm}$ ( $\mathrm{St}>0.12$ for $d=5 \mu \mathrm{m})$.

If every dimension of the computational domain in Fig. 4 is reduced by a factor of 0.5 , which is quite straightforward with a parameterized blockMeshDict file, we obtain $P=0.969$, $0.960,0.954$ and 0.951 for $h=0.5,1.0,1.5$ and $2.0 \mathrm{~mm}$ at $Q=30 \mathrm{ccm}$ (comparable to the case of $Q=120 \mathrm{ccm}$ in Table 5 for similar flow velocity), respectively. At $Q=60 \mathrm{ccm}$, we have $P=0.975$ and 0.970 for $h=1.0$ and $1.5 \mathrm{~mm}$. Even at $Q=6 \mathrm{ccm}$, the value of $P$ for $h=1.0 \mathrm{~mm}$ is still 0.818 and $P=0.811$ for $h=1.5 \mathrm{~mm}$. Inertial impaction of particles on the chamber wall and ceiling could become quite noticeable at $Q=240 \mathrm{ccm}$ ( $\mathrm{St} \sim 0.12$ for $d=5 \mu \mathrm{m}$, especially for $h>1.0 \mathrm{~mm}$ ). Thus, we can come up with a feasible design of mist flow turning chamber with a similar geometry but two different sizes: one for mist flow rate $Q>60 \mathrm{ccm}$ (with $h=2 \mathrm{~mm}$ as in Fig. 4), and the other for $Q<120 \mathrm{ccm}$ (with $h=1.0 \mathrm{~mm}$ and every dimension reduced by half), to adequately cover the mist flow rate range of $5<Q<500 \mathrm{ccm}$ for typical Aerosol Jet $\AA$ printing of features from $\sim 10 \mu \mathrm{m}$ to $\sim 1000 \mu \mathrm{m}(1 \mathrm{~mm})$.

This computational case for the mist flow analysis exemplifies a practical approach for Aerosol Jet ${ }^{\circledR}$ printing system design and optimization, without being bogged down too much by lots of details.

\section{SUMMARY}

To facilitate effective design and optimization of mist transport pathways for the Aerosol Jet ${ }^{\circ}$ direct-write system, a set of multiphase flow models has been established by using both 
analytical formulas, whenever which can be derived, and numerical computations with solvers implemented in the framework of OpenFOAM ${ }^{\circledR}$ CFD Toolbox. The analytical formulas shown in this work for particle trajectory when moving in straight circular tubes allow examination of the nonzero St effects relevant to large particles, extending previous results based on inertialess particle assumption. The results show that the effects of particle inertia can manifest rather complicated trends, due to the interplay of three different dimensionless parameters: St, $U_{s}$ and $\Gamma$. The straight tube analytical model is useful for estimating the mist transport channel length and cross-section area in the Aerosol Jet ${ }^{\circledR}$ system design. For portions with more complex geometries (e.g. the mist flow turning chamber shown in Fig. 4), analytical formulas are difficult to derive and numerical computational models are resorted to. The CFD simulations of mist flow can offer insights into detailed mist particle behaviour and flow structure that are often difficult to visualize with available laboratory instruments. The numerical results, in combination with analysis based on physical principles, can be used for exploring various design concepts and for design optimization, as part of our virtual engineering practice.

\section{ACKNOWLEDGEMENTS}

The author would like to thank Doug Welter for support, and John Hamre, Dr. Mike Renn, as well as many other Optomec colleagues, for valuable technical discussions.

\section{REFERENCES}

[1] Renn, M.J., Direct Write ${ }^{\mathrm{TM}}$ system. US Patent 7,108,894 B2, 2006.

[2] Binder, S., Glatthaar, M. \& Raddlein, E., Analytical investigation of aerosol jet printing. Aerosol Science and Technology, 48(9), pp. 924-929, 2014.

https://doi.org/10.1080/02786826.2014.940439

[3] Feng, J.Q., A computational study of high-speed microdroplet impact onto a smooth solid surface. Journal of Applied Fluid Mechanics, 10(1), pp. 243-256, 2017. https://doi.org/10.18869/acadpub.jafm.73.238.26440

[4] May, K.R., The Collison nebulizer: description, performance and application. Journal of Aerosol Science, 4, pp. 235-243, 1973. https://doi.org/10.1016/0021-8502(73)90006-2

[5] Pich, J., Theory of gravitational deposition of particles from laminar flows in channels. Journal of Aerosol Science, 3, pp. 351-361, 1972. https://doi.org/10.1016/0021-8502(72)90090-0

[6] Wang, C.-S., Gravitational deposition of particles from laminar flows in inclined channels. Journal of Aerosol Science, 6, pp. 191-204, 1975. https://doi.org/10.1016/0021-8502(75)90088-9

[7] Friedlander, S.K., Smoke, dust, and haze, fundamentals of aerosol behavior, John Wiley \& Sons: New York, 1977. https://doi.org/10.1002/aic.690230526 